\title{
Wing Design Optimization of a Solar-HALE Aircraft
}

\section{JaeHoon Lim* and Sun Choi*}

School of Mechanical and Aerospace Engineering, Seoul National University, Gwanak-ro 1, Gwanak-gu, Seoul, Republic of Korea

\section{SangJoon Shin**}

School of Mechanical and Aerospace Engineering/Institute of Advanced Aerospace Technology, Seoul National University, Gwanak-ro 1, Gwanak-gu, Seoul, Republic of Korea

\section{Dong-Ho Lee L** $^{* *}$}

School of Mechanical and Aerospace Engineering, Seoul National University, Gwanak-ro 1, Gwanak-gu, Seoul, Republic of Korea

\begin{abstract}
We develop a preliminary design optimization procedure in this paper regarding the wing planform in a solar-powered highaltitude long-endurance unmanned aerial vehicle. A high-aspect-ratio wing has been widely adopted in this type of a vehicle, due to both the high lift-to-drag ratio and lightweight design. In the preliminary design, its characteristics need to be addressed correctly, and analyzed in an appropriate manner. In this paper, we use the three-dimensional Euler equation to analyze the wing aerodynamics. We also use an advanced structural modeling approach based on a geometrically exact one-dimensional beam analysis. Regarding the structural integrity of the wing, we determine detailed configuration parameters, specifically the taper ratio and the span length. Next, we conduct a multi-objective optimization scheme based on the response surface method, using the present baseline configuration. We consider the structural integrity as one of the constraints. We reduce the wing weight by approximately $25.3 \%$ from that in the baseline configuration, and also decrease the power required approximately $3.4 \%$. We confirm that the optimized wing has sufficient flutter margin and improved static longitudinal/ directional stability characteristics, as compared to those of the baseline configuration.
\end{abstract}

Key words: Design optimization, Solar HALE aircraft, preliminary design

\section{Nomenclature}

$b \quad=$ span length

$c \quad=$ chord distribution

$e \quad=$ total specific energy

$p \quad=$ pressure

$\alpha \quad=$ angle of attack

$\beta \quad=$ sideslip angle

$\bar{c} \quad=$ length of mean aerodynamic chord

$\varepsilon \quad=$ downwash angle

$\sigma \quad=$ sidewash angle $\rho=$ air density

$K \quad=$ kinetic energy density

$U \quad=$ strain energy density

$Q \quad=$ conservative variable vector

$S \quad=$ wing area

$C_{D} \quad=$ drag coefficient

$C_{L} \quad=$ lift coeff icient

$k_{n} \quad=$ empirical wing-body interference factor

$k_{R l} \quad=$ empirical correction factor that is a function of the fuselage Reynolds number

$l_{f} \quad=$ length of the fuselage

$\mathrm{P}_{\text {req }} \quad$ power required
This is an Open Access article distributed under the terms of the Creative Commons Attribution Non-Commercial License (http://creativecommons.org/licenses/by$\mathrm{nc} / 3.0 /$ which permits unrestricted non-commercial use, distribution, and reproduction in any medium, provided the original work is properly cited. (cc) * Graduate Student

** Professor, Corresponding author : ssjoon@snu.ac.kr $* * *$ Emeritus professor 


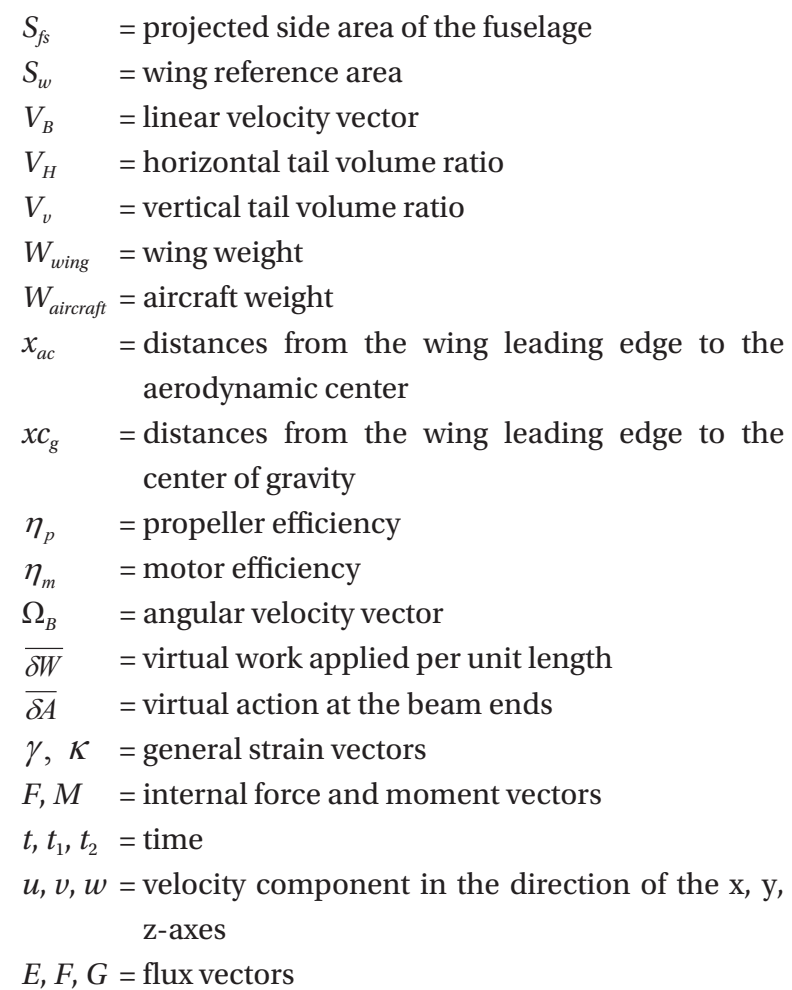

\section{Introduction}

Unmanned aerial vehicles (UAVs) are defined as powered aerial vehicles that do not carry a human operator. They can fly autonomously, or be piloted remotely. The Predator and the Global Hawk are two well-known military UAVs that are currently in operation. During the past 30 years, there has been a significant amount of effort to design and build a UAV that relies on reusable energy. The success of Sunrise I showed that a UAV could be powered by solar energy for high-altitude long-endurance (HALE) missions. Recent solar-powered HALE UAVs, specifically Pathfinder, Centurion, and Helios, have been developed by AeroVironment Inc., with assistance from NASA. Two challenging aspects pertaining to the HALE UAV are determinations of its ceiling altitude and endurance time. Pathfinder was capable of climbing to a maximum altitude of 50,000 ft. Helios was capable of reaching approximately $96,863 \mathrm{ft}$, which is the current world record. The Helios development program came to a halt, when one such UAV encountered extremely high winds during flight, in 2003. That caused excessive natural dihedral, and resulted in a catastrophic crash into the Pacific Ocean [1]. Zephyr, manufactured by QinetiQ, established a world record for the longest endurance, at 14 days and 21 minutes, in 2010 [2]. However, special efforts to design such aircraft are still needed,. The challenge is to design and build a safe, reliable, and efficient solar-powered aircraft, so that disasters such as the Helios mishap do not occur.

UAVs can be classified according to several performance characteristics or specifications, in particular weight, endurance, range, maximum altitude, and wing loading For example, Global Hawk is categorized as belonging to the super-heavy weight class, and features long-endurance, high-altitude, and high wing-loading capabilities. On the other hand, most solar-powered HALE UAVs are classified as belonging to lightweight classes with low wing loadings, but they are still capable of reaching high altitudes, and operating for long times. Here, 'high altitude' signifies that a UAV can climb to 30,000 ft. 'Long endurance' UAVs can be airborne for 24 hours or more. UAVs with a 'low wing loading' have a wing loading of less than $3.12 \mathrm{lb} / \mathrm{ft}^{3}\left(50 \mathrm{~kg} / \mathrm{m}^{3}\right)$.

High-aspect-ratio wings have been widely adopted in long-endurance UAVs, due to both the high lift-to-drag ratio and the light weight. Compared to the wings of conventional fixed-wing aircraft, these types of wings are fairly flexible. In high-aspect-ratio wings, a significant amount of the structural deformation induced from aerodynamic loads will influence the surrounding air particle flow. However, the aerodynamic loads will then be influenced by the structural deformation. Hence in a high-aspect-ratio wing, the interaction between the aerodynamic and structural factors is prevalent. In addition, wing planform design variables, such as the taper ratio and span length, have a significant influence on the aerodynamic performance and weight of an aircraft. Finally, the aeroelastic characteristics of an aircraft with a low wing loading and a flexible high-aspect-ratio wing will differ from those of a conventional wing.

Therefore in the preliminary design stage, the characteristics of solar-powered-HALE (S-HALE) UAVs need to be addressed correctly, and analyzed in an appropriate manner. In the preliminary design stage, the overall system configuration will be defined and modified to satisfy the design requirements. With the results from the preliminary design, a more detailed design will be initiated.

The aircraft design community has recently become heavily dependent upon computational fluid dynamics (CFD) and computational structural mechanics (CSM) analyses, to replace traditional approaches based on simplified theories and wind tunnel tests. Martins demonstrated a new integrated aerodynamic structural design method for aerospace vehicles [3]. They employed high-fidelity models for both aerodynamic and structural disciplines, and used a high-fidelity interaction procedure. However, the object aircraft was a supersonic business jet, which was heavy, and used a low-aspect-ratio wing. 
Moreover, the interaction between aerodynamic and structural factors was not considered. In order to improve the aerodynamic performance and reduce the structural weight for a high-aspect-ratio wing, Long et al. carried out a fluid-structure interaction optimization [4]. Although highfidelity CFD and CSD were used, aeroelastic considerations were not included for the relevant heavy wing. Therefore, it is likely that these would be inappropriate for use with an S-HALE UAV.

Rajagopal et al. $[5,6]$ formulated a preliminary wing design for a low-speed, long-endurance UAV as a two-step optimization problem. Their first step included a singleobjective aerodynamic optimization process, and the second step involved interactive dual-objective aerodynamic and structural optimization. They included a detailed airfoil optimization scheme in their preliminary wing design, and conducted an XFLR5 panel method analysis. However, they considered the span length only as a wing planform design variable. They conducted a structural analysis using MSCNASTRAN. Cestino [7] developed a computer program to carry out a parametric study of the S-HALE UAV platform design. He developed a blended wing body configuration as a result of this parametric study; however, he did not include the nonlinear characteristics due to the high-aspect-ratio structure, and only considered the traditional bendingtorsion flutter in his aeroelastic analysis. In high-aspectratio wings, the potential for single-bending-degree-offreedom flutter is high, and thus needs to be investigated. Previous studies did not consider the interaction between aerodynamic and structural factors and wing planform design variables. If a high-aspect-ratio wing features natural dihedral due to its high lift, its aerodynamic performance and the internal forces/moments acting on the structure will differ. Wing planform design variables have a significant amount of influence on the aerodynamic performance, and the structural integrity may be satisfied by evolution of the design variables. Therefore, preliminary aircraft design optimization processes require wing planform design variables, and an accounting of the interaction between the aerodynamic and structural factors.

The response surface methodology has been widely used to handle various aeromechanical design optimization problems [8-11]. The response surface methodology provides a way to relieve the burden in the integration of large computer programs, by converting it into constructing approximations. The response surface method is used to decouple the analysis and the optimization.

This paper develops a preliminary design optimization procedure regarding the wing planform in an S-HALE UAV, using both high-fidelity aerodynamic and structural analyses. The present optimization procedure will be useful for designing a high-aspect-ratio wing with low wing loading adopted in a lightweight S-HALE UAV, as part of its preliminary design. We use the three-dimensional Euler equation to analyze the wing aerodynamics. We correct drag with the empirical equations, and use an advanced structural modeling approach based on a geometrically exact one-dimensional beam analysis. In addition, we assess the direct interaction between the aerodynamic load and structural deformation.

Considering the structural integrity of the wing, we consider and determine detailed configuration parameters, specifically the composite ply angle, the number of the plies used, and the locations of the spars. We do not perform detailed cross-sectional design optimization, to avoid the associated high computational cost. Moreover, if the detailed cross-sectional design variables are considered in the optimization, the objective function will be highly nonlinear. Next, using the present baseline wing, we conduct a multiobjective optimization scheme based on the response surface method. The use of the response surface method in the present design optimization process serves to reduce the analysis cost. Given that one of the important requirements for current S-HALE UAVs is long-term endurance, the first objective is performance enhancement at high altitude. Also, achieving the minimum structural weight is another requirement for general aircraft designers. Hence, we consider minimization of the wing weight as an additional objective. In the present optimization, we select the taper ratio and the span length, which govern the aerodynamic performance, and the weight of the wing, as the primary design variables. The volume of certain subsystems, such as payload and equipment subsystems, usually determines fuselage sizing and design. Therefore, the only object to be optimized in the present paper is the wing. We consider the structural integrity as a constraint, and use ZAERO software to conduct the aeroelastic stability analysis of the wing [12]. Finally, we also conduct a brief investigation of the aircraft flight dynamic stability.

\section{Optimization Procedure and Analysis Model}

We select the wing configuration of the Zephyr aircraft as the baseline for the present optimization. We obtain this by optimization, based on the energy balancing technique [13]. During the day, this aircraft is required to climb to 23.62 $\mathrm{km}$, in order to convert its extra electric energy to potential energy. Then during the night, it descends to $16.39 \mathrm{~km}$, 
using the potential energy. Table 1 summarizes the aircraft properties [13].

Figure 1 shows a flowchart of the present optimization procedure for an S-HALE UAV. We use a DAE 31 airfoil for the cross-sectional shape of the wing, due to its outstanding performance at low Reynolds number. We use an aerodynamic-structural combined analysis under a cruise flight condition to predict the internal forces and moments at each wing station. We conduct failure analysis using the maximum strain criteria. We determine detailed cross-sectional configuration parameters considering the structural integrity of the wing. Prior to creating experimental design points, we define the allowable ranges of the design variables in terms of the upper and lower bounds. We choose the taper ratio and span length as the design variables, and use them to adjust and determine the wing planform. A $3 \mathrm{k}$ full factorial design method provides enough response points to permit estimations of the response surface coefficients. As a result, we obtain nine experimental points, and conduct an aerodynamicstructural combined analysis at each point. Using the experimental points, we establish a response surface, and determine the optimized design point at which the required power and weight are minimized. Next, we conduct both a flight dynamic stability analysis, and an aeroelastic stability analysis at the optimized design point. If the resulting optimized design point satisfies the stability requirements,

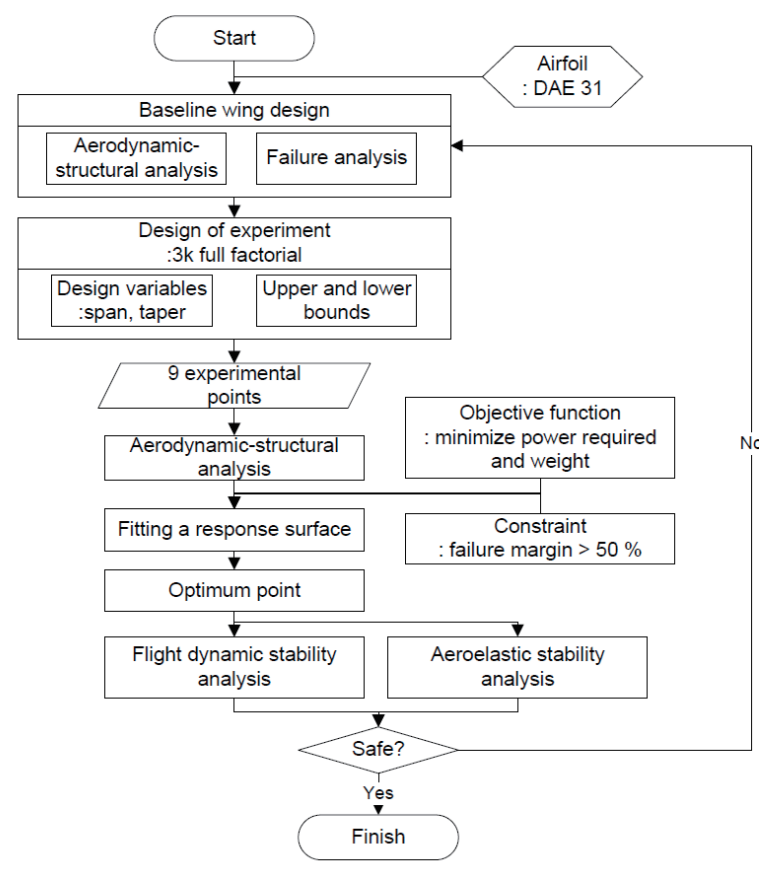

Fig. 1. Present design optimization procedure

the procedure terminates. Otherwise, we repeat the baseline wing cross-sectional design. We perform such an iterative procedure, until all of the results satisfied the required criteria. We explain details about each analysis in the present optimization procedure below.

Table 1. Properties of the object aircraft [13]

\begin{tabular}{ll}
\hline Main wing & \\
\hline Span length (m) & 22.56 \\
Chord (m) & 1.48 \\
\hline Tail wing & 6.08 \\
\hline Span length (m) & 0.72 \\
Chord (m) & \\
\hline Fuselage & 8.2 \\
\hline Fuselage length (m) & 3.42 \\
\hline Weight & 11.76 \\
\hline Solar panel weight (kg) & 6.95 \\
\hline Battery and motor weight (kg) & \\
\hline Fuselage and tails (kg) & 0.1 \\
\hline Operating condition & \\
\hline Maximum speed (Mach number) & \\
\hline
\end{tabular}




\subsection{Aerodynamic Analysis}

We use a high-fidelity CFD algorithm based on the threedimensional Euler equation to predict the aerodynamic forces acting on the wing. The three-dimensional Euler equation can be written in the following non-dimensional conservative form,

$$
\frac{\partial Q}{\partial t}+\frac{\partial E}{\partial x}+\frac{\partial F}{\partial y}+\frac{\partial G}{\partial z}=0
$$

where,

$$
Q=\left(\begin{array}{l}
\rho \\
\rho u \\
\rho v \\
\rho w \\
\rho e
\end{array}\right), E=\left(\begin{array}{l}
\rho u \\
\rho u^{2}+p \\
\rho u v \\
\rho u w \\
(\rho e+p) u
\end{array}\right), F=\left(\begin{array}{l}
\rho v \\
\rho u v \\
\rho v^{2}+p \\
\rho v w \\
(\rho e+p) v
\end{array}\right), G=\left(\begin{array}{l}
\rho w \\
\rho u w \\
\rho v w \\
\rho w^{2}+p \\
(\rho e+p) w
\end{array}\right)
$$

In Eqs. (1) and (2), $Q$ is the conservative variable vector, while $E, F$, and $G$ are flux vectors. $\rho$ is the density, and $u, v$, and $w$ are the velocity components in the $\mathrm{x}, \mathrm{y}$, and $\mathrm{z}$-axis direction, respectively. $e$ is the total specific energy, and $p$ is the pressure.

To discretize the computational domain, we adopt the upwind method. The time integration scheme we adopt in this paper is the LU-SGS (Lower-Upper Symmetric Gauss Seidel) scheme. In addition, we introduce an implicit residual smoothing scheme, in order to accelerate the convergence of the numerical analysis, and reduce the computational time [14]. The drag coefficient obtained by the Euler equation may not be accurate enough. Therefore, we correct it by applying empirical equations [15]. We define the profile viscous drag coefficient, $c_{d p}$, as Eq. (3):

$$
c_{d p}=c_{d 0}+c_{d 2} c_{l}^{2}
$$

where,

$$
c_{d 0}=\frac{K c_{f} S_{\text {wet }}}{S_{\text {ref }}}, \frac{S_{\text {wet }}}{S_{\text {ref }}}=2.04 \text { for Sean's study [16], } c_{d 2}=\frac{0.38}{\cos ^{2} \Lambda} c_{d 0}
$$

In Eq. (4), $K$ is the correction factor for the pressure drag and the increased local velocity, which is a function of the thickness, chord ratio, and wing sweepback angle; while $c_{f}$ is the skin friction coefficient, which is a function of the Reynolds number.

Figure 2 shows the DAE 31 airfoil selected for the crosssectional shape of the wing, because it is well known for its outstanding performance at low Reynolds numbers [16]. In addition, we generate an $\mathrm{O}-\mathrm{H}$ type volume grid, as shown in Figures 3 and 4 . The number of meshes we create is 121 along the airfoil surface. These consist of 33 along the spanwise direction, and 94 normal to the wing surface.

\subsection{Structural Analysis}

To consider the large deflection of the wing in an S-HALE UAV, we apply a nonlinear beam analysis. We then divide the three-dimensional, geometrically nonlinear elastic problem of the beam element into a two-dimensional cross-sectional analysis, and a one-dimensional beam analysis.

\subsubsection{Geometrically Exact Beam Theory}

The nonlinear intrinsic beam formulation originally suggested by Hodges [18] was based on a small amount of strain and large deformation with finite rotation. Shang implemented this formulation in the frequency domain [19]. By adopting this formulation, an analysis of a structure that exhibits large deflection with initial curvature and twist becomes possible, without any geometrically simplifying approximations. We obtain the variational formulation, by applying Hamilton's principle:

$\int_{t_{1}}^{t_{2} l} \int_{0}^{l}[\delta(K-U)+\overline{\delta W}] d x_{1} d t=\overline{\delta A}$

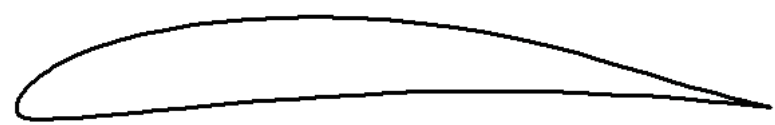

Fig. 2. DAE31 airfoil

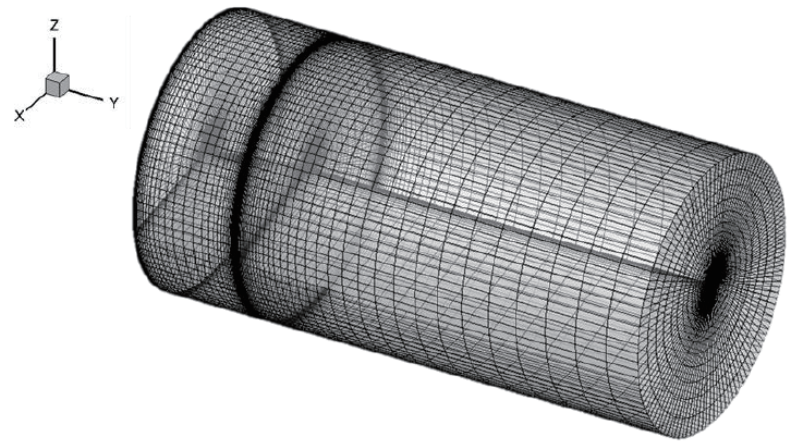

Fig. 3. O-H type volume grid

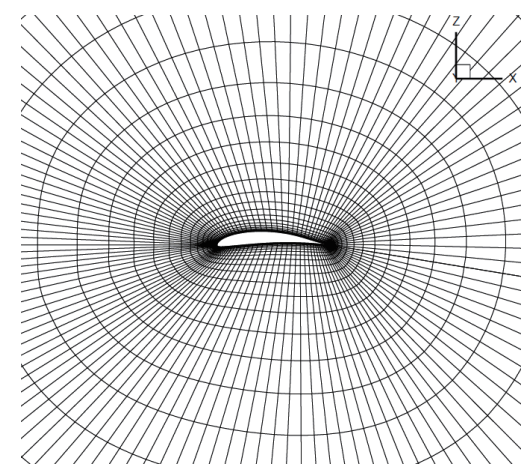

Fig. 4. Plane view of the wing root section 
Here, $t_{1}$ and $t_{2}$ are arbitrarily fixed times, and $K$ and $U$ are the kinetic and strain energy densities per unit length, respectively. $\overline{\delta A}$ is the virtual action at the beam ends, and at the ends of the time interval. In addition, $\overline{\delta W}$ is the virtual work of the applied loads per unit length. $x_{1}$ and $t$ refer to the spanwise coordinate and time, respectively. We formulate the variations of the kinetic and potential energy terms with respect to the arguments $V_{B}$ and $\Omega_{B}$, the linear and angular velocity vectors, and with respect to $\gamma$ and $\kappa$, the general strain vectors. We introduce the internal force and moment vectors $F_{B}$ and $M_{B}$, and the linear and angular momentum vectors $P_{B}$ and $H_{B}$, using the equations below:

$$
F_{B}=\left(\frac{\partial U}{\partial \gamma}\right)^{T}, \quad M_{B}=\left(\frac{\partial U}{\partial \kappa}\right)^{T}, \quad P_{B}=\left(\frac{\partial K}{\partial V_{B}}\right)^{T}, \quad H_{B}=\left(\frac{\partial K}{\partial \Omega_{B}}\right)^{T}
$$

By substituting the above terms into Eq. (3), we obtain:

$$
\int_{i, 0}^{t}\left[\delta \delta V_{B}^{*} P_{B}+\delta \Omega_{B}^{*} H_{B}-\delta \gamma_{B}^{*} F_{B}-\delta \kappa_{B}^{*} M_{B}+\delta F_{B}^{T}\left(\gamma-\gamma^{*}\right)\right] d x_{1} d t+\int_{t_{1}}^{t} \int_{0}^{t} \overline{\delta W} d x_{1} d t=\overline{\delta A}
$$

where, * denotes the degree of satisfaction of the geometrically exact relationships, and where the subscript $B$ indicates the deformed reference frame. We can extract the internal forces and moments directly in the present formulation, because it is a mixed formulation [19]. We use basic linear shape functions for its integration, and obtain accurate predictions using the present mixed form.

\subsubsection{Cross-Sectional Analysis of the Wing}

We combine the present beam formulation with the beam cross-sectional properties, which we express in $6 \times 6$ fully populated mass and stiffness matrices. We adopt a separate cross-sectional analysis to provide these matrices. In the present paper, we use a variational asymptotic beam section analysis (UM/VABS) [20] to analyze the beam cross-sectional configurations in the numerical results. UM/VABS is capable of analyzing a variety of cross-sectional configurations with sufficient accuracy, including closed, open, and thin- and thick-walled sections.

To conduct a structural integrity analysis of the optimized composite wing cross-section, we develop a threedimensional strain recovery module, as shown in Figure

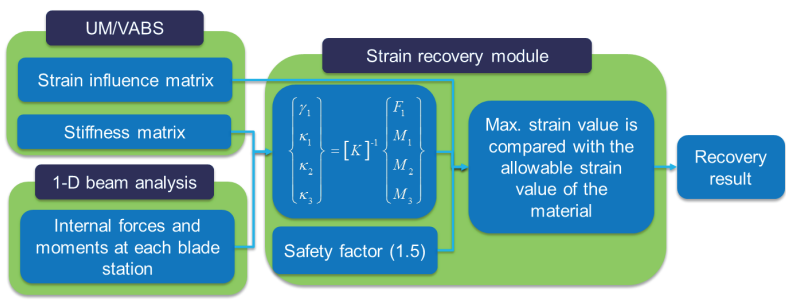

Fig. 5. Flowchart of the three-dimensional strain recovery analysis
5. First, we obtain a strain influence matrix and a stiffness matrix through a UM/VABS. The strain influence matrix is composed of the influence coefficients of the local strain vector with respect to each measured beam deformation. We obtain the local strain vectors upon the reference point of each beam cross-section. Next, using the inverse form of the one-dimensional global beam constitutive relationship, Eq. (8), we obtain the strains and curvatures at a wing section as a function of the internal force and moments.

$$
\left\{\begin{array}{l}
\gamma_{1} \\
\kappa_{1} \\
\kappa_{2} \\
\kappa_{3}
\end{array}\right\}=[K]^{-1}\left\{\begin{array}{l}
F_{1} \\
M_{1} \\
M_{2} \\
M_{3}
\end{array}\right\}
$$

In Eq. (6), $[K]$ is the stiffness matrix that we obtain from UM/VABS. $\gamma_{1}$ is the axial strain, $\kappa_{1}$ is the elastic twist, and $\kappa_{2}$ and $\kappa_{3}$ are the two bending curvatures. We predict the internal forces and moments at each wing section by the present aerodynamic analysis. We multiply the strains and curvatures obtained at a few wing stations by the strain influence matrix for each wing element we obtain from the three-dimensional strain recovery [21]. Each component of the strain influence matrix is the strain corresponding to unit classical deformation. In addition, we consider a safety factor of 1.5. Then, we apply the maximum strain criterion for each component in the resulting strain, and in each case, compare this with the allowable values of the local constituent material.

\subsection{Optimization}

Eq. (9) shows the objective function we establish in the present optimization procedure. The objective function is to minimize the gross weight and the power required, when the aircraft is operating at a medium altitude $(20 \mathrm{~km})$.

$$
\text { minimize } J=\left[w_{1} \frac{W_{\text {wing }}}{W_{\text {wing, baseline }}}+w_{2} \frac{\mathrm{P}_{\mathrm{req}}}{\mathrm{P}_{\mathrm{r} e q, \text { baseline }}}\right]
$$

subject to: failure margin at low operating altitude $\geq 50 \%$

$$
0.5 \leq \text { taper ratio } \leq 1.0,10.152 \leq \text { span } \leq 12.408
$$

For an aircraft in a level flight condition, we can estimate the power required using Eq. (10) [22].

$$
\mathrm{P}_{\mathrm{req}}=\sqrt{\frac{2 W_{\text {aircraft }}{ }^{3}}{\rho S}} \sqrt{\frac{C_{D}{ }^{2}}{C_{L}^{3}}} \eta_{m} \eta_{p}
$$

Here, $\eta_{m}$ is the motor efficiency, and $\eta_{p}$ is the propeller efficiency. In this paper, we assume the motor and propeller efficiencies to be 0.8 and 0.85 , respectively. The crosssectional analysis provides the mass per unit span. We estimate the wing weight by using the mass per unit span, 
taper ratio, and span. We require the resulting wing to have a sufficient failure margin at the critical loading condition. We define the failure margin in Eq. (11).

$$
\text { Failure margin }(\%)=\frac{\text { material allowable strain }- \text { estimated maximum strain }}{\text { material allowable strain }} \times 100
$$

We evaluate the failure margin by the aforementioned strain recovery analysis. At a low altitude $(16.39 \mathrm{~km})$, we predict the internal forces and moments at each wing station by the proposed approach under a maximum cruise flight condition at a Mach number of 0.1. We expect that cruising at low altitude is one of the critical flight conditions for an S-HALE UAV.

The design variables we use in the present optimization procedure are the taper ratio and spanwise length of the wing. We determine the lower bounds by the required wing area to produce enough lift for continuous flight. We apply taper between 50 and $100 \%$ in the span.

We use Jump (JMP) [23], a separate design optimization software package, to build an approximate model, based on the response surface method (RSM). The aforementioned aerodynamic-structural combined analysis requires a significantly large computational time. In order to reduce the computational time for an optimized solution, we adopt RSM. Once we establish the response surface using an experimental technique, we require a relatively short computational time.

Typically, the response surfaces are obtained as polynomials whose orders are identical to the number of the design variables selected. In this paper, we construct a second-order polynomial surface, as Eq. (12) describes:

$$
f(x)=A_{0}+\sum_{i=1}^{n} A_{i} x_{i}+\sum_{i=1}^{n} \sum_{j=1}^{i} A_{i j} x_{i} x_{j}
$$

Here, $A_{0}, A_{i}$ and $A_{i j}$ are the regression coefficients; and $n$ is the number of the design variables selected. We evaluate the fitness of the established approximate model by investigating $\mathrm{R}$ squared, which is the coefficient of determination.

We use a genetic algorithm included in the MATLAB Global Optimization Toolbox [24]. A genetic algorithm, one of the global optimization methods, is a stochastic search technique that imitates the natural evolution process. When a global optimization process is selected, convergence to an objective function generally becomes less sensitive.

\subsection{Aerodynamic-Structural Combined Analysis}

This paper utilizes direct interaction between the aerodynamic load and the structural deflection. The aerodynamic load causes structural deformation, and the structural deformation affects the aerodynamic load.
Thus, the close interaction between the displacement of the deflected wing and the geometry of the aerodynamic grid becomes a required procedure. In this procedure, we assume that the geometry of the airfoil remains unchanged during the structural deflection, as the major deflection of the wing is caused by its own bending behavior.

Figure 6 shows the procedure of the aerodynamicstructural combined analysis. We conduct the aerodynamic analysis using the initially undeformed wing structure. As a result, we obtain the aerodynamic loads, and transfer them to each structural node. Next, we conduct a structural analysis under the relevant load condition. We transfer the translation and rotational angles of the cross-section as predicted by the structural analysis to an aerodynamic analysis. We displace the surface grid, while maintaining each shape of the wing cross-section. Aerodynamic grids cover the deflected shape of the wing, and we regenerate these to compute the aerodynamic loads. To achieve the converged wing deflection, we repeat the previous steps.

The details pertaining to the aerodynamic-structural combined analysis are in the literature [25]. We conduct the aerodynamic-structural analysis using a simple isotropic beam model with the present aerodynamic analysis, for an operating altitude of $15 \mathrm{~km}$, and flight speed of Mach number 0.3. As a result, when the interaction is considered, the lift capability of the present rectangular wing decreases

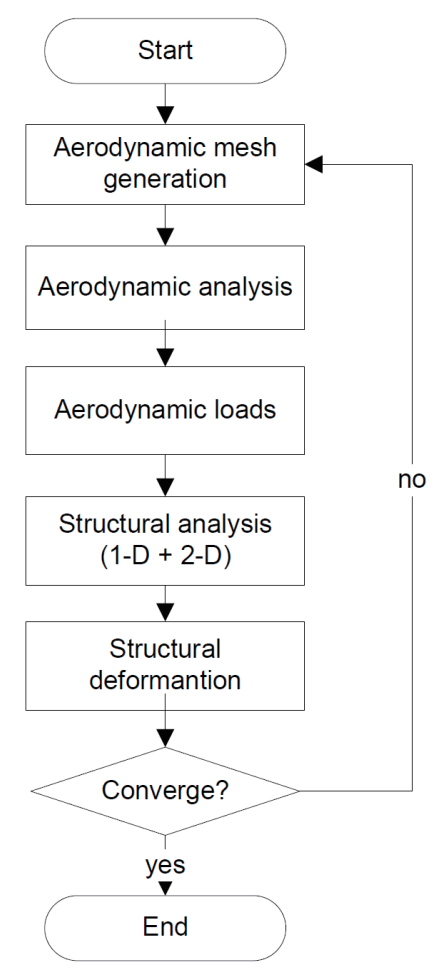

Fig. 6. Flowchart of the present aerodynamic-structural combined analysis 
by approximately $7.12 \%$. The lift coefficient influences the required amount of power, as shown in Eq. (8).

\subsection{Flight Dynamic Stability Analysis}

We separate the aircraft into its individual components, i.e., the wing, fuselage, and tail, to determine its static stability. We sum the contribution of each component, in order to estimate the stability derivative coefficients, as shown in Eq. (13) [26].

$$
\begin{aligned}
& C_{m_{\alpha}}=C_{L_{\alpha_{w}}}\left(\frac{x_{c g}}{\bar{c}}-\frac{x_{a c}}{\bar{c}}\right)-\eta V_{H} C_{L_{\alpha_{t}}}\left(1-\frac{d \varepsilon}{d \alpha}\right) \\
& C_{n_{\beta}}=-k_{n} k_{R l} \frac{S_{f s} l_{f}}{S_{w} b}+V_{v} \eta C_{L_{\alpha_{v}}}\left(1+\frac{d \sigma}{d \beta}\right)
\end{aligned}
$$

In these equations, $k_{n}$ is the empirical wing-body interference factor, and $k_{R l}$ is an empirical correction factor that is a function of the fuselage Reynolds number. We then investigate the variation of the stability derivative coefficients between the baseline wing and the optimized wing.

\subsection{Aeroelastic Stability Analysis}

We conduct an aeroelastic stability analysis of the optimized wing using ZAERO software [12], which is based on the doublet lattice method. In order to obtain the mode shapes and natural frequencies of the optimized wing, we use the aforementioned three-dimensional, geometrically nonlinear beam analysis. Within ZAERO, we estimate the unsteady aerodynamics, based upon the structural mode shapes. In more detail, we apply two-dimensional panel aerodynamics to the corresponding beam model. The S-HALE UAV performs its mission at high altitude. Therefore, we need to investigate the flutter speed variation with respect to the altitude. We estimate the flutter speed of the present optimized wing using the g-method provided in ZAERO. The detailed formulation of the g-method is available in the literature [12].

\section{Numerical Results}

\subsection{Preliminary Cross-Sectional Design of the Base- line Wing}

We determine the cross-sectional design parameters of the baseline wing by maintaining the failure margin at more than $50 \%$ at all spanwise stations. Figure 7 illustrates the crosssection design result. We use T300/5208 graphite-epoxy for the spars, and Mylar polyester film for the skin. We locate the forward spar at $0.22 c$, and its rear counterpart at $0.32 c$. The ply orientation angles of the spar are $[0 / 45 /-45 / 0]_{s}$. The ply orientation angles of the spar caps are identical to those of the spars. We use a symmetric ply stack sequence for the spar caps and the spars. The ply orientation angle of the skin is $0^{\circ}$. We adjust the numbers of plies for the spar and spar caps to maintain the structural failure requirement. By conducting a strain recovery analysis, we find that the maximum strain occurs at the wing root section, and the failure margin is $52.7 \%$.

In this paper, we do not include cross-sectional design variables in the design variables, due to the ensuing high computational cost when doing so. However, we plan to develop a separate design optimization procedure by including those design variables. If we consider the detailed cross-sectional design variables in the optimization, the objective function will be highly nonlinear. In such a case, we will use the Kriging model, as it is appropriate for approximate highly nonlinear functions.

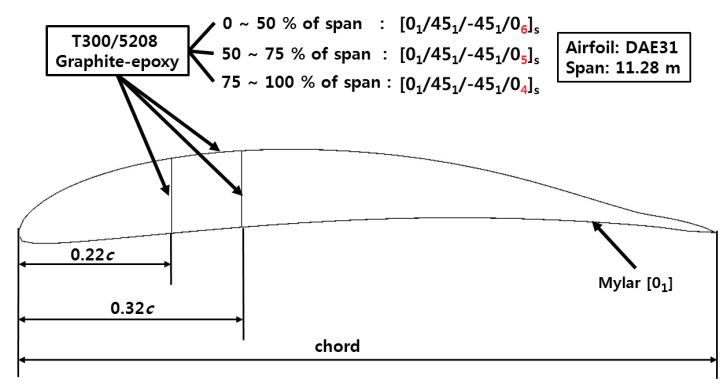

Fig. 7. Cross s ectional configuration of the present UAV wing

\subsection{Optimization}

Using the determined baseline wing structure, we conduct the aerodynamics-structural combined analysis at nine experimental points, and construct response surfaces. We construct the second-order response surface for the objective function, as shown in Eq. (14).

$$
\begin{aligned}
f(\text { taper, span })= & 1.8344+0.1163 \text { taper }+0.0705 \text { span } \\
& +0.0509 \text { taper }^{2}+0.0092 \mathrm{taper} \cdot \mathrm{span}+0.0072 \mathrm{span}^{2}
\end{aligned}
$$

In addition to the objective function, we construct the response surfaces for the wing weight, required power, and failure margin. To investigate the appropriateness of the present response surface model, we conduct a regression analysis, Table 2 shows these results. The coefficients of determination for the wing weight, the required power, and the objective function are larger than 0.99; these are indicated by the letter ' $a$ ' in Table 3 . This guarantees the reliable prediction capability of the present response surface. However, the coefficient of determination for the failure margin, indicated by the letter ' $b$ ' in Table 3 , is smaller 
than 0.99 , due to the fact that the spanwise location of the element is the point where the resulting maximum strain will change as the taper ratio and span length vary. Therefore, we determine that a failure analysis of the optimized wing is necessary, to verify its structural integrity.

Table 2. Results of the present regression analysis

\begin{tabular}{cc}
\hline & $\begin{array}{c}\mathrm{R}^{2} \\
\text { (coefficient of determination) }\end{array}$ \\
\hline Objective function & $0.9996^{\mathrm{a}}$ \\
Wing weight & $0.9998^{\mathrm{a}}$ \\
Power required & $0.9989^{\mathrm{a}}$ \\
Failure margin & $0.9826^{\mathrm{b}}$ \\
\hline
\end{tabular}

Figure 8 shows the variation of the response surfaces with respect to the taper ratio and span length. As the taper ratio and span length decrease, the objective function also decreases. The wing weight shows a trend similar to that of the objective function. In contrast, a wing with a longer span has an advantage with regard to the required power. For a constant failure margin, we show that in terms of the structural integrity, a lower span and less tapered wings are favorable.

In order to obtain the optimized values of the weighting factors, pre-defined in Eq. (7) as $w_{1}$ and $w_{2}$, we construct a Pareto front for the present objective function. Table 3 summarizes the present values of the objective function and design variables for the present optimization. When $w_{1}$ is larger than 0.4 , we maintain the resulting values of the objective function and design variables. Therefore, we divide the weightings equally to both objective functions as 0.5 .

Table 4 summarizes the optimized values of the design variables, and Figure 9 illustrates the optimized configuration. The taper ratio of the optimized wing is 0.5 , and its span length is 10.152 . Using the optimized design variables, we reduce the wing weight by approximately $25.3 \%$, compared to that in the baseline condition, as Table

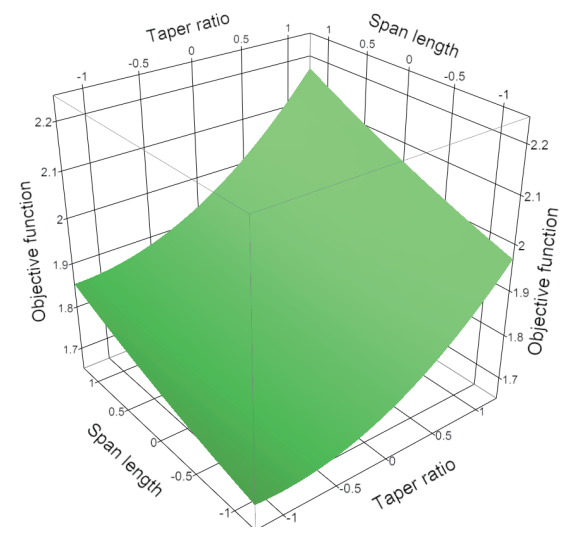

(a) objective function

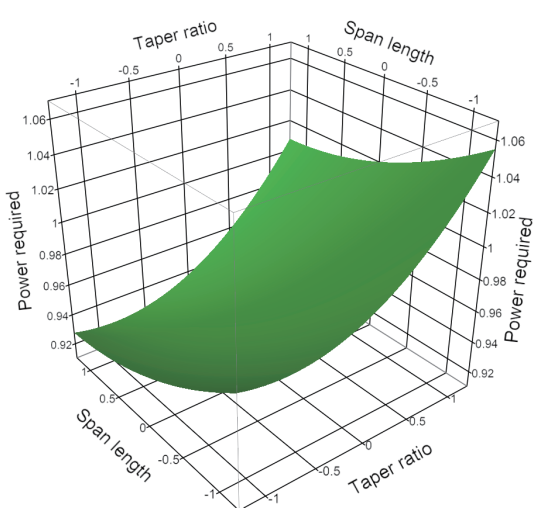

(c) power required at altitude of $20 \mathrm{~km}$

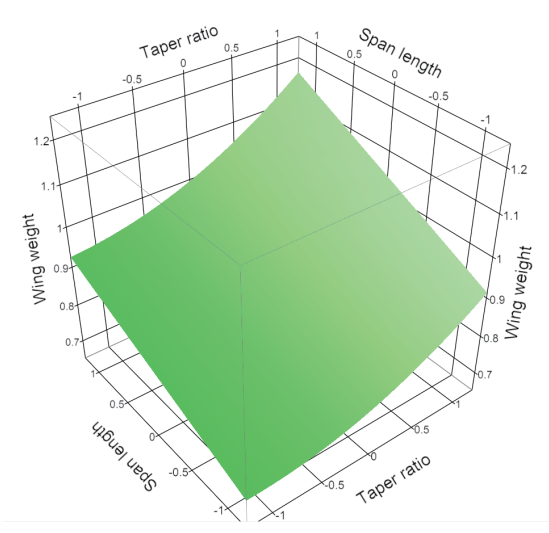

(b) wing weight

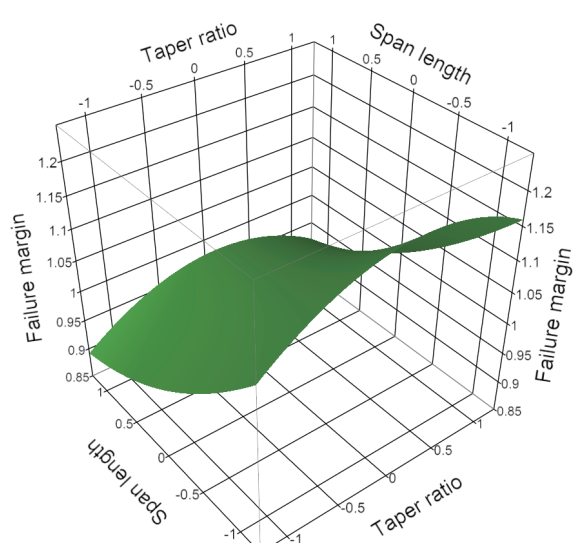

(d) failure margin

Fig. 8. Response surface created for the present UAV wing, in terms of the taper ratio and span length 
Table 3. The resulted values of the objective function and design variables for the present optimization

\begin{tabular}{cccccc}
\hline $\begin{array}{c}\text { Weighting } \\
\text { factor (1) }\end{array}$ & $\begin{array}{c}\text { Weighting } \\
\text { factor (2) }\end{array}$ & $\begin{array}{c}\text { Objective } \\
\text { function (1) }\end{array}$ & $\begin{array}{c}\text { Objective } \\
\text { function (2) }\end{array}$ & $\begin{array}{c}\text { Design variable } \\
(1)\end{array}$ & $\begin{array}{c}\text { Design variable } \\
(2)\end{array}$ \\
\hline$w_{1}$, & $w_{2}$ & $\frac{W_{\text {wing }}}{W_{\text {wing,baseline }}}$ & $\frac{\mathrm{P}_{\text {req }}}{\mathrm{P}_{\text {req, baseline }}}$ & Taper ratio & Span length (m) \\
\hline 0.1 & 0.9 & 0.8968 & 0.9272 & 0.5192 & 12.1732 \\
0.2 & 0.8 & 0.8310 & 0.9391 & 0.5083 & 11.2891 \\
0.3 & 0.7 & 0.7482 & 0.9671 & 0.5091 & 10.1520 \\
0.4 & 0.6 & 0.7477 & 0.9674 & 0.5039 & 10.1525 \\
0.5 & 0.5 & 0.7478 & 0.9673 & 0.5001 & 10.1522 \\
0.6 & 0.4 & 0.7477 & 0.9674 & 0.5000 & 10.1520 \\
0.7 & 0.3 & 0.7478 & 0.9673 & 0.5002 & 10.1521 \\
0.8 & 0.2 & 0.7477 & 0.9674 & 0.5000 & 10.1520 \\
0.9 & 0.1 & 0.7477 & 0.9674 & 0.5004 & 10.1524 \\
\hline
\end{tabular}

Table 4. Results of the design variables in both the baseline and optimized configurations

\begin{tabular}{ccc}
\hline Design variable & Baseline & Optimized \\
\hline Taper ratio & 1 & 0.5 \\
Span length $(\mathrm{m})$ & 11.28 & 10.152 \\
\hline
\end{tabular}

5 shows. In addition, we decrease the required power by approximately $3.4 \%$. However, this increases the failure margin by approximately $5.1 \%$. As mentioned previously, a failure analysis is necessary in the optimized wing to verify its structural integrity. But it is not necessary in this

Baseline wing

Optimized wing

Fig. 9. Optimized wing configuration case, because the optimized design point is one of the nine experimental points.

In this paper, we determine the airfoil shapes prior to the optimization task. In addition to the planform design optimization process, we can conduct airfoil geometry optimization to improve the aerodynamic performance of the aircraft.

\subsection{Flight Dynamic Stability Analysis}

Table 6 illustrates the $C_{m_{a}}$ and $C_{m_{\beta}}$ values used for the baseline, and in the optimized configuration. Given that the wing contribution to directional stability is quite small,

Table 5. Results of the wing weight and power required in both the baseline and optimized configurations

\begin{tabular}{|c|c|c|c|}
\hline & \multicolumn{2}{|c|}{ Objectives } & \multirow{2}{*}{$\begin{array}{c}\text { Constraint } \\
\text { Failure margin }(\%)\end{array}$} \\
\hline & Wing weight $(\mathrm{kg})$ & $\begin{array}{l}\text { Power required at altitude of } 20 \\
\mathrm{~km} \text { (watt) }\end{array}$ & \\
\hline Baseline & 50.56 & 937.18 & 55.1 \\
\hline Optimized & 37.75 & 905.7 & 50.0 \\
\hline Difference (\%) & -25.3 & -3.4 & +5.1 \\
\hline
\end{tabular}


in comparison to that of the tail, the coefficients are zero. To ensure longitudinal static stability, the aircraft must have a negative value of $C_{m_{a}}$. Also, the yawing moment coefficient versus the sideslip angle beta for the aircraft must be positive for static directional stability. According to the table, we expect that the optimized configuration will have better static longitudinal and directional stability characteristics than both of the baseline aircraft.

\subsection{Aeroelastic Stability Analysis}

We conduct an aeroelastic stability analysis of the optimized wing with ZAERO software, and investigate the flutter speed variation with respect to the altitude. Table 7 shows this result. Single-bending-degree-of-freedom flutter takes place, rather than the traditional bending-torsion flutter. This occurs regardless of the altitude, and the flutter speed increases with respect to the altitude. However, flutter does not occur under the maximum operating condition at Mach number $0.1(34 \mathrm{~m} / \mathrm{s})$. The predicted flutter margin at ground level is approximately $102 \%$. Therefore, we confirm that the optimized wing has sufficient flutter stability. Moreover, the optimized wing shows increased torsional rigidity, as a $45^{\circ}$ ply orientation angle is used for the spars and spar caps, as shown in Figure 7. Thus, we expect that the traditional bending-torsion flutter will not occur. We conduct an additional flutter analysis with a different wing, using ply orientation angles of only 0 and $90^{\circ}$, in an effort to investigate the possibility of traditional bending-torsion flutter. As a result, bending-torsion flutter occurs when the aircraft flies at an altitude of $16.39 \mathrm{~km}$ or higher, as shown in Table 8.

\section{Conclusions}

In this paper, we develop a wing planform design optimization procedure for an S-HALE UAV, using both high-fidelity aerodynamic and structural analyses. We use the three-dimensional Euler equation to analyze the wing aerodynamics, and use an advanced structural modeling approach based on a geometrically exact one-dimensional

Table 6. Flight dynamic stability characteristics of both the baseline and optimized configurations

\begin{tabular}{cccc}
\hline & & $C_{m_{\alpha}}$ & $C_{n_{\beta}}$ \\
\hline \multirow{2}{*}{ Wing } & Baseline & 0.258 & 0 \\
& Optimized & 0.258 & 0 \\
\multirow{2}{*}{ Tail } & Baseline & -0.923 & 0.094 \\
& Optimized & -1.074 & 0.124 \\
& Baseline & -0.665 & 0.094 \\
Total & Optimized & -0.817 & 0.124 \\
& &
\end{tabular}

Table 7. Results of aeroelastic stability analysis for the optimized wing

\begin{tabular}{ccl}
\hline Altitude $(\mathrm{km})$ & Flutter speed $(\mathrm{m} / \mathrm{s})$ & Flutter mode \\
\hline 0 & 69.2 & Single degree of freedom flutter (Bending) \\
10 & 134.9 & Single degree of freedom flutter (Bending) \\
16.39 & 138.5 & Single degree of freedom flutter (Bending) \\
20.00 & 131.5 & Single degree of freedom flutter (Bending) \\
23.62 & 157.1 & Single degree of freedom flutter (Bending) \\
\hline
\end{tabular}

Table 8. Results of aeroelastic stability analysis for a different wing (only including 0 or $90^{\circ}$ ply orientation angles)

\begin{tabular}{ccc}
\hline Altitude $(\mathrm{km})$ & Flutter speed $(\mathrm{m} / \mathrm{s})$ & Flutter mode \\
\hline 0 & 45.43 & Single degree of freedom flutter (Bending) \\
10 & 96.39 & Single degree of freedom flutter (Bending) \\
16.39 & 137.9 & Bending-torsion flutter \\
20.00 & 172.3 & Bending-torsion flutter \\
23.62 & 214.2 & Bending-torsion flutter \\
\hline
\end{tabular}


beam analysis for a flexible high-aspect-ratio wing. We determine detailed configuration parameters considering the structural integrity of the wing. Next, we conduct multiobjective optimization based on the response surface method, using the obtained baseline wing. We consider the structural integrity as one of the constraints. As a result, we reduce the wing weight by approximately $25.3 \%$, compared to that in the baseline configuration; and also decrease the required power by approximately $3.4 \%$. We assess the aeroelastic stability analysis of the optimized wing with ZAERO software, and conduct a brief investigation of the aircraft flight stability. Single-bending-degree-of-freedom flutter occurs at a much higher speed than the maximum operating condition of the baseline aircraft. We confirm that the optimized wing has sufficient flutter margin and improved static longitudinal/directional stability characteristics, as compared to the baseline configuration.

In the future, we will consider additional design variables related to the airfoil geometry and detailed cross-sectional design variables for a composite wing in the present design optimization procedure. If we consider the detailed crosssectional design variables in the optimization scheme, the objective function will be highly nonlinear. In such a case, we will use the Kriging model, as it is suitable for approximating highly nonlinear functions. By considering additional design variables, we expect to obtain further improvements of the aerodynamic performance, and a reduction in the weight.

\section{Acknowledgments}

This work was supported by Advanced Research Center Program (NRF-2013R1A5A1073861) through the National Research Foundation of Korea(NRF) grant funded by the Korea government(MSIP) contracted through Advanced Space Propulsion Research Center at Seoul National University. This work was also supported by a grant (No. 20124030200030) of the Human Resources Development program, of the Korea Institute of Energy Technology Evaluation and Planning (KETEP), funded by the Korea government Ministry of Trade, Industry and Energy.

\section{References}

[1] Najafi, Y., "Design of a High Altitude Long Endurance Solar Powered UAV, M. S. Thesis, San Jose State University, 2011.

[2] Anonymous, "Breakthrough Unmanned Aerial Surveillance Technology, Zephyr," Qinetiq, 2009.
[3] Martines, J. R. R. A., and Alonso, J. J., "Complete Configuration Aero-structural Optimization Using Coupled Sensitivity Analysis Method," $9^{\text {th }}$ AIAA/ISSMO Symposium on Multidisciplinary Analysis and Optimization, Atlanta, GA, USA, September 4-6, 2002.

[4] Long, T., Liu, L., Li, Y., Peng, L., Huang, B., and Qi, Z., "Aero-structure Coupled Optimization of High Aspect Ratio Wing Using Enhanced Adaptive Response Surface Method," $12^{\text {th }}$ AIAA ATIO Conference and 14th AIAA/ISSM Indianapolis, India, September, 17-19, 2012.

[5] Rajagopal, S., and Ganguli, R., "Multidisciplinary Design Optimization of Long Endurance Unmmaned Aerial Vehicle Wing," Computer Modeling in Engineering and Science, Vol. 81, No. 1, 2001, pp.1-34.

[6] Rajagopal, S., and Ganguli, R., "Conceptual Design of UAV using Kriging based multi-objective genetic algorithm," Aeronautical Journal, Vol. 112, No. 1137, 2008, pp. 653-662.

[7] Cestino, E., "Design of Solar High Altitude Long Endurance Aircraft for Multi payload and Operations," Aerospace Science and Technology, Vol. 10, No. 6, 2006, pp. 541-550.

[8] Viswamurthy, S., R., and Ganguli, R., “Optimal Placement of Trailing-edge Flaps for Helicopter Vibration Reduction using Response Surface Methods," Engineering Optimization, Vol. 39, No. 2, 2007, pp. 185-202.

[9] Sobiesky, I.. P., and Kroo, I. M., "Collaborative Optimization using Response Surface estimation," AIAA Journal, Vol. 38, No. 10, 2000, pp. 1931-1938.

[10] Senthil, M., and Ganguli, R., "Aeroelastic Stability Enhancement and Vibration Suppression in a Composite Helicpopter Rotor," Journal of Aircraft, Vol. 42, No. 4, 2005, pp. 1013-1024.

[11] Keane, A. J., "Wing Optimization using Design of Experiment, Response Surface, and Data Fusion Method," Journal of Aircraft, Vol. 40, No. 4, 2003, pp. 741-750.

[12] Anonymous, ZAERO Version 8.4 Theoretical Manual, ZONA Technology Inc., Scottsdale, AZ, USA, 2008.

[13] Park, K., Min, S., Ahn, J., and Lee, D.-H., "Multidisciplinary Design Optimization (MDO) of a Medium-Sized Solar Powered HALE UAV Considerring Energy Balancing," KSAS, Vol. 40, No. 2, 2012, pp. 129-138.

[14] Kim, Y.-S., "Multidisciplinary Multi-Point Design Optimization of Supersonic Fighter Wing," Ph. D. Dissertation, Department of Aerospace Engineering, Seoul National University, 2003.

[15] Wakayama, S., "Lifting Surface Design Using Multidisciplinary Optimization," Ph. D. Dissertation, Department of Aeronautics and Astronautics, Stanford University, 1994.

[16] Langford, J., “The Daedalus Project : A Summary of 
Lessons Learned", AIAA/AHS/ASEE Aircraft Design, Systems and Operations Conference, 1989, pp. 89-2048

[17] Shevell, R. S., Fundamentals of Flight, $2^{\text {nd }}$ edition., Prentice-Hall, Englewood Cliffs, NJ, 1989.

[18] Hodges, D. H., "A Mixed Variational Formulation Based on Exact Intrinsic Equations for Dynamics of Moving Beams," International Journal of Solids and Structures, Vol. 26, No. 11, 1990. pp. 1253-1273.

[19] Shang, X., "Aeroelastic Stability of Composite Hingeless Rotors with Finite-State Unsteady Aerodynamics," Ph.D. thesis, Georgia Inst. of Technology, Atlanta, GA, USA, Aug. 1995.

[20] Palacios, R. and Cesnik, C. E. S., "Cross-Sectional Analysis of Non-Homogeneous Anisotropic Active Slender Structures," AIAA Journal, Vol. 43, No. 12, 2005, pp. 2624-2638.

[21] Shin, S.-J., Cesnik, C. E. S., Wilkie, W. K., and Wilbur,
M. L., "Design and Manufacturing of a Model-scale Active Twist Rotor Prototype Blade", Journal of Intelligent Material Systems and Structures, Vol.19, No.12, 2008, pp. 1443-1456.

[22] Anderson, J., Fundamentals of Aerodynamics, fifth edition, Mcgraw Hill, New yYorkcity, year2010.

[23] Anonymous, JMP User Guide, Release 8, SAS Institute Inc. 2009.

[24] Anonymous, Global Optimization Toolbox Reference Version 3, Mathworks, Inc, 2004.

[25] Choi, S., Park, K., and Lee, D.,-H., "Aerodynamic Loss of HALE Aircraft Wing Due to Large Deflection Considering Various Wing Configurations," 2012 Asia-Pacific International Symposium on Aerospace Technology, Jeju, Korea, Nov. 13-15, 2012.

[26] Nelson, R. C., Flight Stability and Automatic Control, $2^{\text {nd }}$ edition, Mcgraw-Hill, New yYorkcity, 1998. 